\title{
Systematic review of interventions to increase the delivery of preventive care by primary care nurses and allied health clinicians
}

Kathleen M. McElwaine ${ }^{1,2,3,5^{*}}$, Megan Freund ${ }^{1,2,3}$, Elizabeth M. Campbell ${ }^{1,2,3}$, Kate M. Bartlem ${ }^{1,3,4}$, Paula M. Wye $\mathrm{e}^{1,3,4}$ and John H. Wiggers ${ }^{1,2,3}$

\begin{abstract}
Background: Primary care nurses and allied health clinicians are potential providers of opportunistic preventive care. This systematic review aimed to summarise evidence for the effectiveness of practice change interventions in increasing nurse or allied health professional provision of any of five preventive care elements (ask, assess, advise, assist, and/or arrange) for any of four behavioural risks (smoking, inadequate nutrition, alcohol overconsumption, physical inactivity) within a primary care setting.

Methods: A search of Medline, Embase, Psyclnfo, and CINAHL databases was undertaken to locate controlled intervention trials published between 1992 and May 2014 that provided practice change interventions to primary care nurses and/or allied health professionals to increase preventive care. The effect of interventions aimed at increasing the provision of any of the five care elements for any of the four behavioural risks was examined. A narrative synthesis was utilised.
\end{abstract}

Results: From 8109 articles, seven trials met the inclusion criteria. All trials bar one, assessed multi-strategic practice change interventions (three to five strategies) focused on care by nurses (six trials) or mixed nursing/allied health clinicians. One trial examined care provision for all four risks, five trials examined care for smoking only, and one trial examined care for alcohol consumption only. For the six trials reporting significance testing (excludes one smoking care trial), significant effects favouring the intervention group were reported in at least one trial for smoking risk assessment (2/4 trials reported an effect for at least one analysis of an assessment outcome), brief advice (2/3), assistance (2/2), and arranging referral (2/3); alcohol risk assessment (1/2) and brief advice (1/2); inadequate nutrition risk assessment (1/1); and physical inactivity risk assessment and brief advice (1/1). When the number of analyses undertaken within trials focusing on smoking care was considered, the results were less promising (e.g. of the 15 analyses conducted on brief advice variables across three trials, four showed a positive effect).

Conclusions: Evidence for the effect of practice change interventions on preventive care by primary care nurses or allied health providers is inconclusive given the small number of trials and inconsistency of results between and within trials.

Systematic review registration number: None

Keywords: Prevention and control, Primary health care, Review, Systematic

\footnotetext{
* Correspondence: Kathleen.McElwaine@newcastle.edu.au

${ }^{1}$ Population Health, Hunter New England Local Health District, Booth

Building, Wallsend Health Services, Longworth Avenue, Wallsend, NSW 2287,

Australia

${ }^{2}$ Faculty of Health, The University of Newcastle, University Drive, Callaghan,

NSW 2308, Australia

Full list of author information is available at the end of the article
} 


\section{Background}

The routine delivery of primary preventive care by primary health care providers is recommended by national and international clinical guidelines [1-8] to reduce the disease burden caused by four priority risk behaviours: tobacco smoking, inadequate nutrition, alcohol overconsumption, and physical inactivity [9-11]. Primary health care clinicians encompass a variety of health care professionals such as nurses and allied health professionals including physiotherapists, dieticians, and occupational therapists, among others [12-16].

Such guidelines recommend that preventive care is provided by a range of primary health care providers including nurses (e.g. practice nurses, nurses, midwives) $[1-3,5]$ and allied health clinicians [5, 7] (e.g. health visitors, physiotherapists, exercise professionals, and health trainers) [5]. Preventive care is recommended to be provided for multiple risks [1, 2], routinely and opportunistically when clients present for reasons not necessarily related to their preventable health risk behaviours [1-8]. Five preventive care elements are recommended to meet this guidance: asking all patients about the four behavioural risks (risk assessment); assessment of readiness to change and dependence (for smoking and alcohol); brief, nonjudgemental advice with patient educational materials and motivational interviewing; assistance by providing motivational counselling and pharmacotherapy if required (for nicotine or alcohol dependence); and arrangement of a referral to telephone support services, group lifestyle programs, or an individual provider (e.g. dietician), and a follow-up visit where applicable $[17,18]$. These elements are commonly referred to as the 5A's [1].

Cochrane systematic review evidence supports the effectiveness of preventive care interventions involving elements included within the 5A's approach in modifying the four priority risk behaviours [19-23]. A large proportion of the component studies tested interventions containing elements included within the 5A's, provided by health care providers in a variety of health care settings, predominantly primary care settings [19-23]. Taken together with additional individual studies and non-Cochrane reviews undertaken in primary care settings specifically, the evidence for the effectiveness of preventive care is strongest for smoking cessation [24-28], and to a lesser extent alcohol overconsumption $[14,18,27,29-31]$, with accumulating evidence for inadequate nutrition $[14,18,24,27$, $32]$ and physical inactivity [14, 18, 24, 33, 34]. While brief preventive care interventions appeared to have modest behaviour change impacts, and typically only a minority of those receiving an intervention may make clinically significant changes in risk behaviour, such an effect translates to significant health benefits at the population level when systematically applied to the large proportion of people that are at risk [14].

Within the primary care setting, nurses and allied health clinicians have the potential to be key providers of preventive care $[12,14,17,18,35-39]$ as their care focuses on chronic disease prevention and management $[12,36]$, often delivered on multiple occasions to population groups with a high prevalence of behavioural risks [12, 36, 40, 41].

Despite the potential of primary care nurses and allied health clinicians to provide preventive care, variable levels of its provision have consistently been reported internationally in primary care practices [42-52]. For example, in a study in the UK, 30-50\% of primary care nurses reported they actively addressed smoking, inadequate nutrition, alcohol overconsumption, or physical inactivity with a large proportion of their clients [53]. An Australian study using client report found generalist community nurses and allied health clinicians provided brief advice for these four behavioural risks to between $43 \%$ and $66 \%$ of clients at risk [39]. A further Australian study [54] based on client self-report of care provision by nursing and allied health clinicians (which encompassed psychologists/psychiatrists/counsellors, social workers, occupational therapists, physiotherapists, and dieticians/nutritionists, among others) [55] found the prevalence of clinician assessment to not exceed $60 \%$ for any of four behavioural risks; only $16 \%$ of clients were assessed for all four risks; and referral/followup was offered to less than $5 \%$ of clients for individual risks and to less than $1 \%$ for all four risks combined [54]. Such data suggests there is a need to increase the delivery of preventive care by primary care nurses and allied health clinicians.

Cochrane reviews have examined the effectiveness of practice change interventions in improving the delivery of health care practices generally (including preventive care delivery, test ordering/utilisation, prescribing, management of a presenting problem, data recording, and diagnosis), delivered primarily by physicians in settings that included primary care [56-61]. All such reviews found practice change intervention strategies were effective in producing small to moderate improvements in the delivery of the specified health care practice. The reviews focused on the impact of the following practice change strategies: educational meetings [56], educational outreach visits and academic detailing [61], professional, financial and organisational interventions [57], audit and feedback [58], printed educational materials [59], and financial incentives [60]. Conclusions regarding the effectiveness of the utilisation of multiple of the above intervention strategies are limited by each review examining one particular type of intervention strategy. 
Three systematic reviews have examined the effect of practice change strategies on delivery of preventive care for smoking [62], or alcohol consumption [63, 64] within primary care settings, with the clinicians targeted in the included studies being predominantly primary care physicians. The review on smoking care supported the effectiveness of single strategy interventions (including performance feedback, reminders and prompts, academic detailing) on some elements of care. However, it found multi-strategic interventions, defined as interventions combining two or more intervention strategies [62], to be more consistently effective [62]. The alcohol reviews also demonstrated the effect of practice change interventions: one review concluded that both educational and office-based interventions could be effective, resulting in an absolute increase of between $8 \%$ and $18 \%$, with interventions that combine both strategies being most effective [63], while the second review found that alcohol screening and counselling increased with the amount of clinician training and/or support provided; however, the overall effectiveness was modest [64].

No systematic reviews could be located that examined the effectiveness of practice change strategies in increasing the delivery of preventive care specifically by primary care nurses and/or allied health professionals regarding any of four behavioural risks.

\section{Objectives}

Given no systematic reviews have examined the effectiveness of practice change interventions in increasing primary care nurses and/or allied health professionals provision of recommended elements of preventive care for any of the four priority behavioural risks, a systematic review following PRISMA guidelines was undertaken that aimed to summarise such evidence. The current review included controlled intervention trials conducted in a primary care setting that assessed the effect of single or multi-strategic practice change interventions on preventive care provision by nurses and/or allied health professionals. Outcomes of interest were the provision of any of the five recommended elements of preventive care (ask, assess, advise, assist, or arrange) [17] for at least one of four behavioural risks (smoking, inadequate nutrition, alcohol overconsumption, or physical inactivity). Preventive care outcomes for each of the five care elements for the four risks were summarised for control and intervention groups.

\section{Methods}

\section{Eligibility criteria}

Information sources and search strategy

A search of Medline, Embase, PsycInfo, and CINAHL databases was undertaken using the following MeSH headings:
('Primary Health Care' or 'Community Health Centers' or 'Community Health Services' or 'Community Health Nursing'; or 'Attitude of Health Personnel'); and ('Smoking' or 'Smoking Cessation' or 'Alcoholism' or 'Exercise' or 'Diet' or 'Preventive Health Services/og [Organization \& Administration]' or 'Risk Factors'). The search was limited to articles published in the last 20 years, from January 1992 to 2012, and subsequently updated to be current as at May 2014.

\section{Trial selection}

All titles and abstracts retrieved by electronic searching were downloaded into a reference management database (Reference Manager v12), screened by the first author, and studies not meeting the inclusion criteria were excluded. Where not possible to exclude articles based on title and abstract, full text versions were obtained and their eligibility was assessed by the first author. The reference lists of included trials were checked for further relevant trials.

\section{Inclusion criteria}

Identified articles were examined to determine whether the following inclusion criteria were met. Each paper was assessed starting from the first criterion onwards and recorded as excluded on the first criterion it did not meet. Once excluded, the paper was not assessed against subsequent inclusion criteria.

(1) It was in English.

(2) It was a journal article (excluded grey literature such as transcribed interviews, case studies, commentaries, thesis dissertations, reflections, conference abstracts/posters).

(3) It was not a study protocol, review, or editorial.

(4) It quantitatively described at least one of five preventive care outcomes (ask, assess, advise, assist, or arrange) for at least one of the four risks (smoking, inadequate nutrition [including inadequate fruit and vegetable consumption], alcohol overconsumption, or physical inactivity). In an attempt for the search to be more inclusive, 5A's terminology was not required and could be inferred by the extractor based on definitions of the 5A's $[17,18]$. Operational definitions of risk behaviours were not pre-specified but rather were dependent on how each trial defined such risk behaviours. Preventive care outcomes could include measures relating to clients receiving care and/or health professionals providing care.

(5) The preventive care targeted clients who were adults 18 years and older, or the citation reported care data for adults separately to children. 
(6) It was in a primary care setting (including general practice, community health services, Health Maintenance Organisations, Primary Care Trusts, mobile nursing services, medical centre outpatient, university clinic, and dentistry settings). Studies excluded were those set in inpatient and outpatient hospital settings, emergency departments, and residency clinics.

(7) Preventive care was provided by routine staff members as part of routine primary care delivery, not by staff specifically employed to implement preventive care as part of the research (e.g. research assistants).

(8) It was an intervention trial that tested the effect of any practice change strategies (single or multi-strategic interventions) on preventive care provision outcome/s, and that included comparison with a control group (including controlled trials, time series, or controlled before-after trials).

(9) It included nurses or allied health professionals as the practice change intervention target. Allied health professionals included any person involved in the delivery of care (professional or not, regulated or not) that was not a nurse, midwife, or physician.

(10) It reported preventive care outcome data for nurses or allied health professionals. If other types of clinicians were involved (e.g. general practitioners, doctors, residents), the results specifically for the nurses or allied health professionals were available.

\section{Data extraction and description of trials}

Data extraction was undertaken by the first author and recorded into a form which had been developed prior to the search and piloted. Accuracy of extraction was confirmed by a second author checking the data extraction of all variables and studies. Selected trials were summarised alphabetically and described in terms of the following: author, year published, country undertaken in, trial design, trial risk-factor focus, care setting, sample size, practice change intervention strategies utilised, clinician target group, data collection tool, preventive care practices examined, and outcome measures. A narrative synthesis was utilised. A meta-analysis was not planned as it was anticipated that studies would be too heterogeneous to provide a meaningful summary in relation to participants (e.g. clinicians or clients), interventions, and outcomes (e.g. the various health risk behaviours and care elements examined and the potential for multiple analyses to be conducted for each care element within each risk behaviour) [65].

\section{Practice change intervention strategies utilised}

Intervention strategy classification was based on definitions outlined by the Cochrane Effective Practice and
Organisation of Care Group (Table 1). All strategies included in intervention and control group conditions were listed for each trial.

\section{Risk of bias}

To provide an indication of the methodological quality of studies, risk of bias was independently assessed by three review authors (at the outcome level of relevance to the current review; KM, KB, and PW) using the tool outlined in the Cochrane Handbook for Systematic Reviews of Interventions (Additional file 1) [65]. Discrepancies were resolved by consensus among all reviewers and a fourth review author (MF). Sources of bias assessed were those attributable to generation of the random sequence, allocation concealment, blinding of participants and personnel, blinding of outcome assessors, completeness of outcome data, selective reporting, and any other potential threats to validity. Trial authors were contacted via email (including a follow-up email to non-responders) to obtain further information regarding unclear classifications. Results were described narratively.

\section{Practice change intervention effect on preventive care delivery}

The following outcome data for each trial were summarised: clinician provision (prevalence, means and standard deviations, and Likert scores) of any of the five elements of preventive care (ask, assess, advise, assist, or arrange) [17] with regard to the four behavioural risks (smoking, inadequate nutrition, alcohol overconsumption, or physical inactivity). All types of outcome data were summarised (e.g. clinician or client self-report, medical records audit, observations). Follow-up levels of care for control and intervention groups for each trial were described (baseline levels were reported when available), along with results of significance testing.

\section{Results}

\section{Trial selection}

In total, 8109 citations were extracted from the search (see Fig. 1 for PRISMA flow diagram). After duplicates were removed $(n=367), 7742$ abstracts and titles were reviewed. Of these, 7735 articles were excluded as the following: two were not in English; 18 were not journal articles; 7189 were a study protocol, review, or editorial, or did not quantitatively describe the proportion of health professionals providing, or clients provided with preventive care for at least one of the four risks; 25 were based on data related to care for children; 102 were not in a primary care setting; 16 described care that was not provided by routine staff; 308 were not intervention trials or did not have a control/comparison group; 52 did not include nurses or allied health professionals as a 
Table 1 Intervention strategies to change health professional practice ${ }^{a}$

\begin{tabular}{|c|c|}
\hline Interventions & Definition \\
\hline Distribution of educational materials & $\begin{array}{l}\text { Published or printed recommendations for clinical care including clinical } \\
\text { practice change guidelines, delivered personally or through mass mailings. }\end{array}$ \\
\hline Educational meetings & $\begin{array}{l}\text { Health care providers who have participated in conferences, lectures, workshops, } \\
\text { or traineeships. }\end{array}$ \\
\hline Local consensus processes & $\begin{array}{l}\text { Inclusion of participating providers in discussion to ensure that they agreed } \\
\text { that the chosen clinical problem was important and the approach to managing } \\
\text { the problem was appropriate. }\end{array}$ \\
\hline Educational outreach visits and academic detailing & $\begin{array}{l}\text { Use of a trained person who met with providers in their practice settings to give } \\
\text { information with the intent of changing the provider's practice. The information } \\
\text { given may have included feedback on the performance of the provider(s). }\end{array}$ \\
\hline Local opinion leaders & Use of providers nominated by their colleagues as 'educationally influential'. \\
\hline Patient mediated interventions & $\begin{array}{l}\text { New clinical information (not previously available) collected directly from patients } \\
\text { and given to the provider. }\end{array}$ \\
\hline Audit and feedback & $\begin{array}{l}\text { Any summary of clinical performance of health care over a specified period } \\
\text { of time. The summary may also have included recommendations for clinical action. } \\
\text { The information may have been obtained from medical records, computerised } \\
\text { databases, or observations from patients. }\end{array}$ \\
\hline Reminders & $\begin{array}{l}\text { Patient or encounter specific information, provided verbally or on paper, or on a } \\
\text { computer screen, which is designed or intended to prompt a health professional to } \\
\text { recall information, including computer-aided decision support. }\end{array}$ \\
\hline Marketing & $\begin{array}{l}\text { A survey of targeted providers to identify barriers to change and subsequent design } \\
\text { of an intervention that addresses identified barriers. }\end{array}$ \\
\hline Professional & $\begin{array}{l}\text { Individual behaviour (distributing educational materials) and organisational } \\
\text { interventions (local consensus processes). }\end{array}$ \\
\hline Financial & $\begin{array}{l}\text { Includes individual and organisational incentives and environmental restructuring } \\
\text { (changing the available products). }\end{array}$ \\
\hline Organisational & $\begin{array}{l}\text { Includes input (changing skill mix), processes (communication), and effects } \\
\text { (satisfaction of providers). Influencing the organisation of services, including the } \\
\text { process of care (delegation of tasks), the structure of care (the follow-up system), } \\
\text { and the content of care (health charts, flow sheets). }\end{array}$ \\
\hline Regulatory & Includes legal (changes in patient liability) and social influence (peer review). \\
\hline Patient resources ${ }^{\mathrm{b}}$ & $\begin{array}{l}\text { Distribution or addition of resources that may aid discussions of risk factors, or allow } \\
\text { previously unavailable options for preventive care, including flipcharts, educational } \\
\text { resources for patients, and referral opportunities (e.g. quitlines). }\end{array}$ \\
\hline Ongoing support ${ }^{\mathrm{b}}$ & $\begin{array}{l}\text { Email, telephone, or face-to-face communications which provided support and } \\
\text { advice, responded to questions, or problems. }\end{array}$ \\
\hline
\end{tabular}

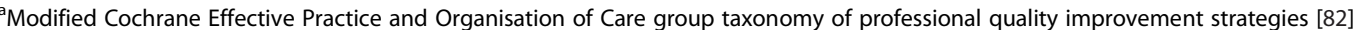

${ }^{b}$ Intervention strategies not covered by EPOC criteria

clinical target of the practice change intervention; and 23 did not report results for nurses or allied health professionals separately from other types of clinicians (e.g. medical practitioners). Consequently, seven trials were included in the current review (Table 2) [66-71]. No additional trials were identified from reference lists.

\section{Trial characteristics}

\section{Year, country, and trial design}

All seven trials were published between 1998 and 2013 (one later than 2004); two were undertaken in the USA $[69,71]$, three in the UK $[68,70,72]$, one in Australia [67], and one in the Netherlands [66]. Four trials were randomised controlled trials [68-70, 72]; one was a cluster randomised controlled trial [66], one was a non-randomised controlled trial [71], and one was a quasi-experimental design [67].

\section{Primary care setting and sample size}

Trials were conducted in primary care practices/ clinics/general practices [68-72], prenatal care clinics [66], and generalist community nursing services [67]. The number of practices ranged from 4 to 212 [66-70, 72]. For the four trials reporting sample sizes for clients, these ranged from 556 to 4048 clients [66, 69, $70,72]$. For the three trials reporting sample sizes for clinicians involved in outcome assessment, these ranged from 30 to 129 nurses $[66,67,71]$ and 80 to 97 allied health clinicians [71]. 


\section{PRISMA 2009 Flow Diagram}

From: Moher D, Liberati A, Tetzlaff J, Altman DG, The PRISMA Group (2009). Preferred Reporting Items for Systematic Reviews and Meta-Analyses: The PRISMA Statement. PLoS Med 6(6): e1000097. doi:10.1371/journal.pmed1000097. For more information, visit www.prisma-statement.org.

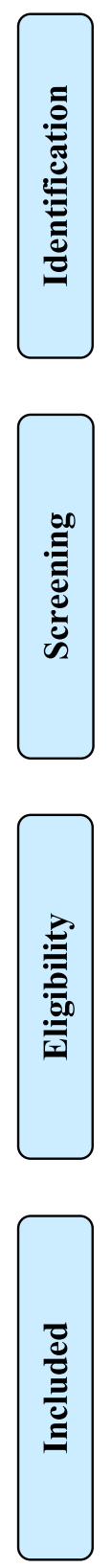

$$
\begin{aligned}
& \text { Records identified through database } \\
& \text { searching } \\
& (\mathrm{n}=8109)
\end{aligned}
$$

Additional records identified through other sources

$$
(\mathrm{n}=0)
$$

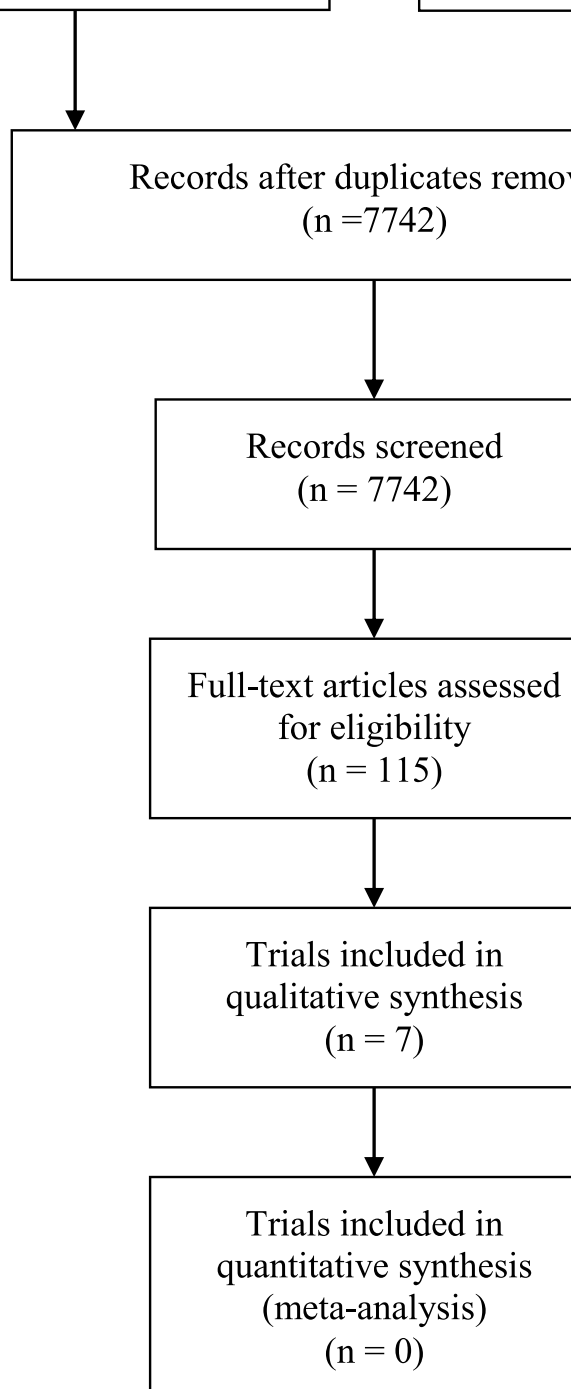

Fig. 1 PRISMA 2009 flow diagram 
Table 2 Intervention trials reporting change in routine provision of preventive care: January 1992-May 2014

\begin{tabular}{|c|c|c|c|c|}
\hline $\begin{array}{l}\text { Author/year/country/ } \\
\text { trial design }\end{array}$ & Trial focus/care setting/sample size & Intervention strategies & Clinician group/data collection tool & $\begin{array}{l}\text { Preventive care practices examined/ } \\
\text { outcome }\end{array}$ \\
\hline $\begin{array}{l}\text {-Bakker et al. (2003) [66] } \\
\text {-Netherlands } \\
\text {-Cluster RCT }\end{array}$ & $\begin{array}{l}- \text {-Smoking cessation for pregnant women. } \\
-42 \text { pre-natal care clinics }(22 \text { IV, } 20 \text { C) } \\
118 \text { midwives: } \\
- \text { IV } n=57 \text { (37 did questionnaire-65 \%) } \\
-C n=61(32 \text { did questionnaire- } 52 \%) \\
556 \text { clients: } \\
- \text { IV } n=253 \text { (sample for presented analysis }=44) \\
-C n=303 \text { (sample for presented analysis }=51 \text { ) }\end{array}$ & $\begin{array}{l}\text { IV: } \\
\text {-Distribution of educational } \\
\text {-Offered educational meetings } \\
\text {-Patient resources } \\
\text { C: } \\
\text {-Patient resources }\end{array}$ & $\begin{array}{l}\text { Clinician target: } \\
\text {-Midwives } \\
\text {-Clinician questionnaire at follow-up. } \\
\text { Client questionnaires. } \\
\text { [M (SD), C vs IV } \\
\text {-Scale } 1-5 \text { for clinician self-report data } \\
(1=\text { never, } 5=\text { always). } \\
\text {-Scale } 0-1 \text { for client self-report } \\
(0=\text { no, } 1=\text { yes). } \\
\text { Continuous variables. }\end{array}$ & $\begin{array}{l}\text { Ask: } \\
\text {-Clinician: } 5.00 \text { vs } 4.91(0.37)^{\mathrm{a}} \\
\text {-Client: } 0.72(0.29) \text { vs } 0.91(0.18)^{* *} \\
\text { Advise: (to quit) } \\
\text {-Clinician: } \\
\text { (during pregnancy): } 4.19(1.03) \text { vs } 4.60(0.77)^{*} \\
\text { (to partner): } 3.58(1.42) \text { vs } 4.03(1.33)^{\mathrm{a}} \\
\text {-Client } \\
\text { (during pregnancy): } 0.64(0.36) \text { vs } 0.85(0.25)^{* *} \\
\text { (post-partum): } 0.04(0.08) \text { vs } 0.21(0.25)^{* *} \\
\text { Assist: } \\
\text {-clinician: } 1.63(1.10) \text { vs } 3.63(1.19)^{* * *} \\
\text {-client: } 0.03(0.16) \text { vs } 0.33(0.34)^{* * *} \\
\text { Arrange: } \\
\text {-clinician: } 2.84(0.99) \text { vs } 3.97(0.89)^{* * *} \\
\text { Results similar when analysed at practice } \\
\text { level (except clinician reported advice to quit } \\
\text { during pregnancy no longer statistically } \\
\text { significant). }\end{array}$ \\
\hline $\begin{array}{l}\text {-Chan et al. (2013) [67] } \\
\text {-Australia } \\
\text {-Quasi-experimental design }\end{array}$ & $\begin{array}{l}\text {-Relevant risks: Smoking, nutrition, alcohol } \\
\text { consumption, and physical inactivity } \\
\text { Other risks: weight } \\
-4 \text { generalist community nursing services } \\
\text { randomised to: } \\
- \text { IV: } n=N R \\
- \text { C: } n=N R \\
\text { Clinicians: } \\
- \text {-Baseline: } n=129 / 178(72.5 \%) \\
-6 \text { months F/U: } n=81 / 129(62.8 \%) \\
-12 \text { months F/U: } n=65 / 129(50.4 \%) \\
\text { Overall response rate across all time } \\
\text { points } n=54 / 129(41.9 \%)\end{array}$ & $\begin{array}{l}\text { IV (early IV): } \\
\text {-Educational meetings } \\
\text {-Reminders } \\
\text {-Patient resources } \\
\text {-Distribution of educational } \\
\text { materials } \\
\text { C (late IV): } \\
\text {-Usual care } \\
\text {-Followed by intervention after } \\
\text { collection of outcome data }\end{array}$ & $\begin{array}{l}\text { Clinician target: } \\
\text {-Generalist community health nurses } \\
\text {-Clinician questionnaire: } \\
\text {-Baseline } \\
-6 \text { months F/U } \\
-12 \text { months F/U } \\
\text { [Group mean effect size ( } 95 \% \text { CI) at } \\
6 \text { and } 12 \text { months F/U, respectively. } \\
\text { Based on Likert scale } 1 \text { (never)- } 7 \\
\text { (always) provided care as part of } \\
\text { routine practice]. } \\
\text { [For ask and advise/assist scores: } \\
\text { tested for significant differential } \\
\text { change between IV vs C groups over } \\
\text { time (baseline, } 6 \text { and } 12 \text { mths F/U). } \\
\text { (Time by group interaction p value). } \\
\text { For Arrange scores: significance } \\
\text { testing conducted for IV vs C groups } \\
\text { at baseline vs } 6 \text { mths, and baseline vs } \\
12 \text { mths] }\end{array}$ & 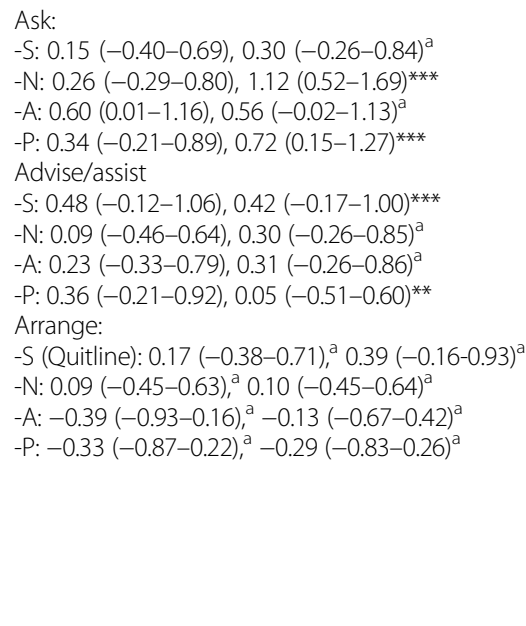 \\
\hline $\begin{array}{l}\text {-Kaner et al., (2003) [68] } \\
\text {-UK } \\
\text {-Cluster RCT }\end{array}$ & $\begin{array}{l}\text {-Alcohol } \\
-212 \text { general practices randomised to: } \\
- \text { C: } n=76 \\
\text {-IV } 1: n=68 \\
\text {-IV } 2: n=68 \\
\text { (data for } 156 \text { practices) }\end{array}$ & $\begin{array}{l}\text { IV 1-Outreach Training } \\
\text {-Educational outreach visit } \\
\text {-Patient mediated intervention } \\
\text {-Educational meeting } \\
\text { IV 2-Training plus telephone- } \\
\text { based support } \\
\text {-Educational outreach visit } \\
\text {-Patient mediated intervention } \\
\text {-Educational meeting } \\
\text {-Ongoing support }\end{array}$ & $\begin{array}{l}\text { Clinician target: } \\
\text {-Nurses } \\
\text { Clinician questionnaire: } \\
\text {-Baseline } \\
-3 \text { months F/U (collection of } \\
\text { screening forms) } \\
\text { (Median [interquartile range], } \\
\text { C vs IV } 1 \text { vs IV 2) }\end{array}$ & $\begin{array}{l}\text { Ask: } \\
0 \text { [0-17] vs } 11[0-28] \text { vs } 13[0-37]^{* *} \\
\text { Advise: } 0[0-3] \text { vs } 1[0-4] \text { vs } 1[0-7]^{*} \\
\text { (\% of clients) } 60 \text { vs } 61 \text { vs } 64 \%^{*}\end{array}$ \\
\hline
\end{tabular}

-Australia

er risks: weight

randomised to:

points $n=54 / 129$ (41.9\%)

-Ongoing support 
Table 2 Intervention trials reporting change in routine provision of preventive care: January 1992-May 2014 (Continued)

\begin{tabular}{|c|c|c|c|c|}
\hline & & $\begin{array}{l}\text { C: } \\
\text {-Patient mediated intervention } \\
\text {-Distribution of educational } \\
\text { materials }\end{array}$ & & \\
\hline $\begin{array}{l}\text {-Katz et al. (2004) [69] } \\
\text {-USA } \\
\text {-RCT }\end{array}$ & $\begin{array}{l}\text {-Smoking cessation } \\
-9 \text { primary care clinics (routine } \\
\text { non-emergency care: } 7 \text { family practice, } \\
2 \text { internal medicine) } \\
\text {-Patients: } n=1221 \text { (includes patients seen by } \\
\text { medical assistants) } \\
n=663 \text { patients seen by below clinicians: } \\
\text { IV sites: } \\
\text {-Registered nurses: } n=100 \\
\text {-Licenced practical nurses: } n=154 \\
\text { C sites: } \\
\text {-Registered nurses: } n=153 \\
\text {-Licenced practical nurses: } n=256\end{array}$ & $\begin{array}{l}\text { IV (clinician targeted): } \\
\text {-Educational meeting } \\
\text {-Audit and feedback } \\
\text {-Reminders } \\
\text {-Patient resources } \\
\text { C: } \\
\text {-Unspecified }\end{array}$ & $\begin{array}{l}\text { Clinician target: } \\
\text {-Registered nurses, } \\
\text {-Licenced practical nurses } \\
\text {-(Medical assistants) } \\
\text { Client interviews (during IV period) } \\
\text { (\% C vs IV site patients receiving care) }\end{array}$ & $\begin{array}{l}\text { Ask: } \\
\text {-Registered nurses: } 67 \% \text { vs } 92 \%{ }^{\mathrm{b}} \\
\text {-Licenced practical nurses: } 35 \% \text { vs } 86 \%{ }^{\mathrm{b}} \\
\text { Assess: } \\
\text {-Registered nurses: } 15 \% \text { vs } 85 \% \%^{\mathrm{b}} \\
\text {-Licenced practical nurses: } 8 \% \text { vs } 75 \%{ }^{\mathrm{b}} \\
\text { Advise: } \\
\text {-Registered nurses: } 16 \% \text { vs } 41 \%{ }^{\mathrm{b}} \\
\text {-Licenced practical nurses: } 7 \% \text { vs } 46 \%{ }^{\mathrm{b}} \\
\text { Assist: } \\
\text {-Registered nurses: } 17 \% \text { vs } 73 \%{ }^{\mathrm{b}} \\
\text {-Licensed practical nurses: } 8 \% \text { vs } 69 \%{ }^{\mathrm{b}}\end{array}$ \\
\hline $\begin{array}{l}\text {-Lennox et al., (1998) [72] } \\
\text {-UK } \\
\text {-RCT }\end{array}$ & $\begin{array}{l}\text {-Smoking } \\
\text {-Primary care: } \\
16 \text { general practices: } \\
\text { (IV, } n=8 ; C, n=8) \text {. } \\
\text { Clinicians receiving IV: } \\
\text { Practice nurses: } 15 / 16(93.7 \%) \\
\text { Health visitors: } 16 / 16(100 \%) \\
\text { Clients: } \\
14 \text { months F/U response rate: } \\
1693 / 2588 \text { (65.4 \%). }\end{array}$ & $\begin{array}{l}\text { IV: } \\
\text {-Educational meeting } \\
\text { C: } \\
\text {-Usual care (no educational } \\
\text { meeting) }\end{array}$ & $\begin{array}{l}\text { Clinician target: } \\
\text {-Practice nurses } \\
\text { - Health visitors } \\
\text {-(General practitioners) } \\
\text { Client questionnaire: } \\
\text { - } 14 \text { months F/U } \\
\text { (\% (n) of C vs IV patients) }\end{array}$ & $\begin{array}{l}\text { Ask: } \\
\text { Practice nurses: } 76.2 \%(n=77 / 101) \\
\text { vs } 83.2 \%(n=104 / 125)^{\mathrm{a}} \\
\text { Health visitors: } 68.6 \%(n=24 / 35) \\
\text { vs } 73.7 \%(n=28 / 38)^{\mathrm{a}}\end{array}$ \\
\hline $\begin{array}{l}\text {-Moher et al., (2001) [70] } \\
\text {-UK } \\
\text {-Cluster } \\
\text { RCT }\end{array}$ & $\begin{array}{l}\text {-Coronary heart disease } \\
\text { Relevant risks: smoking } \\
\text { Other risks: blood pressure, cholesterol } \\
\text {-Primary care: } \\
21 \text { general practices: } \\
\text { (IV } 1, n=7 ; \text { IV } 2, n=7 ; \text { C, } n=7 \text { ). } \\
\text { Clients: } n=4048 \\
\text { Baseline (IV } 1, n=772 ; \text { IV } 2, n=747 ; C, n=623) \text {. } \\
\text { F/U: (IV } 1, n=682 ; \text { IV } 2, n=665 ; C, n=559)\end{array}$ & $\begin{array}{l}\text { Nurse targeted IV: } \\
\text {-Audit and feedback } \\
\text {-Local consensus processes } \\
\text {-Educational outreach visits } \\
\text { and academic detailing } \\
\text {-Ongoing support } \\
\text {-Patient mediated intervention } \\
\text { C: } \\
\text {-Audit and feedback } \\
\text {-Usual care }\end{array}$ & $\begin{array}{l}\text { Clinician target: } \\
\text {-Nurses } \\
- \text {-(General practitioners) }^{c} \\
\text { Medical records audit: } \\
\text {-Baseline } \\
-18 \text { months F/U } \\
\text { [Mean \% of clients (range), C vs } \\
\text { general practitioners targeted IV } \\
\text { vs nurse targeted IV] }\end{array}$ & $\begin{array}{l}\text { Ask: *** } \\
\text {-Baseline: } 73 \%(50-91) \text { vs } 71 \%(47-96) \\
\text { vs } 71 \%(46-85) \\
\text {-F/U: } 78 \%(56-92) \text { vs } 92 \%(77-100) \\
\text { vs } 95 \%(88-98)\end{array}$ \\
\hline $\begin{array}{l}\text {-Secker-Walker et al., (2000) [71] } \\
\text {-USA } \\
\text {-Non-RCT }\end{array}$ & $\begin{array}{l}\text {-Smoking in women (18-64 years) } \\
\text {-Primary care: } n=4 \\
2 \text { IV counties } \\
2 \text { C counties } \\
\text { Clinicians: } n=289 \text { (eligible) } \\
\text {-Dentists } \\
\text { (IV: } n=51, C: n=46), \\
\text {-Dental hygienists } \\
\text { (IV: } n=38, C: n=44), \\
\text {-Family planning counsellors and WIC nurse } \\
\text { counsellors } \\
\text { (IV: } n=14 \text { C: } n=16),\end{array}$ & $\begin{array}{l}\text {-IV (4-year multi-strategic, clin- } \\
\text { ician targeted): } \\
\text { Family Planning and WIC } \\
\text { nurse counsellors: } \\
\text {-Educational meeting } \\
\text {-Patient resources } \\
\text { Dentists, and dental hygienists: } \\
\text {-Educational meeting } \\
\text {-Ongoing support } \\
\text { Mental health counsellors: } \\
\text { (no formal approaches, but } \\
\text { educational meeting attended }\end{array}$ & $\begin{array}{l}\text { Clinician target: } \\
\text {-dentists } \\
\text {-dental hygienists } \\
\text {-family planning counsellors and WIC } \\
\text { nurse counsellors } \\
\text { Clinician questionnaire: } \\
\text {-Baseline } \\
\text {-Yr } 5 \text { F/U } \\
\text {-Yr } 7 \text { F/U } \\
\text { (Means for baseline vs F/U } \\
\% \text { of smokers receiving cessation } \\
\text { activity on } 4 \text { point scale: } 0=\text { none, }\end{array}$ & $\begin{array}{l}\text { Paired Comparisons (Yr 5) } \\
\text { Advise (to quit): } \\
\text {-Dentists: IV: } 1.7 \text { vs } 1.77^{\text {d }} \text { C: } 1.3 \text { vs } 1.6^{\text {a }} \\
\text { Assist (provision of self-help materials): } \\
\text {-Dentists: IV: } 0.2 \text { vs } 0.6 ; \text { C: } 0.3 \text { vs } 0.3^{*} \\
\text {-Dental hygienists: IV: } 0.3 \text { vs } 0.7 ; \text { C: } 0.4 \text { vs } 0.4^{a} \\
\text { Arrange (Referral to support group): } \\
\text {-Dental hygienists: IV: } 0.2 \text { vs } 0.6 ; \\
\text { C: } 0.1 \text { vs } 0.11^{* * *} \\
\text { Arrange (Referral to quit group): } \\
\text {-Dentists: IV: } 0.1 \text { vs } 0.4 ; \text { C: } 0.2 \text { vs } 0.2 ; \\
\text {-Dental hygienists: IV: } 0.3 \text { vs } 0.9 ;\end{array}$ \\
\hline
\end{tabular}


Table 2 Intervention trials reporting change in routine provision of preventive care: January 1992-May 2014 (Continued)

$\begin{array}{ll}\text {-Community mental health counsellors } & \text { by } 3 \text { staff members) } \\ \text { (IV: } n=57, C: n=23) & \text { C: } \\ \text {-Physicians } & \text {-Usual care } \\ \text { (IV: } n=73, C: n=73), & \end{array}$

1 = some, $2=$ most, $3=$ all). (Time by group interaction $p$ value).

Paired comparisons at Yr 5 (Paired)

(IV: $n=73, C: n=73)$

(Means for $C$ vs IV counties $\%$ of

smokers receiving cessation activity

on a 4 point scale: $0=$ none

1 = some, 2 = most, 3 = all)

Unpaired comparisons at Yr 5; Unpaired

comparisons at Yr 7 (Unpaired)

C: 0.3 vs $0.4 ; * *$

Unpaired Comparisons ( $Y r 5$ and $Y_{r} 7$ )

Assist (provision of self-help materials):

-Family Planning and WIC counsellors:

Yr 5: 1.1 vs $1.77^{* *}$ Yr $7: 1.5$ vs $1.6^{\circ}$

Assist (setting quit dates):

-Family planning and WIC counsellors: Yr 5: 0.4

vs $0.8 i^{*}$ Yr $7: 0.7$ vs $0.6^{a}$

Arrange (referral to support group):

-Dentists: Yr 5: 0.0 vs $0.2^{*}$ Yr $7: 0.2$ vs $0.2^{a}$

-Dental hygienists: Yr 5: 0.1 vs 0.6:***

Yr 7: 0.2 vs $0.4^{\mathrm{a}}$

-Family planning and WIC counsellors: Yr 5: 0.4

vs $1.3^{* * * *}$ Yr $7: 0.3$ vs $0.3^{a}$

-Community mental health counsellors:

Yr 5: 0.1 vs $0.44^{*}$ Yr $7: 0.3$ vs $0.3^{a}$

Arrange (referral to quit group):

-Dentists: Yr 5: 0.2 vs $0.4 ;^{*}$ Yr 7: 0.3 vs $0.4^{a}$

Dental hygienists: Yr 5: 0.4 vs 0.9; ** Yr 7: 0.3

vs $0.5^{\mathrm{a}}$

-Family planning and WIC counsellors: Yr 5: 0.7

vs $1.7 i^{* * *} \operatorname{Yr} 7: 1.0$ vs $0.6^{\mathrm{a}}$

Arrange (referral one-to-one telephone support):

-Family planning and WIC counsellors: Yr 5: 0.3

vs $1.4 * * * *$ Yr $7: 0.5$ vs $0.6^{2}$

${ }^{*} p \leq 0.05,{ }^{* *} p \leq 0.01,{ }^{* * *} p \leq 0.001$

$R C T$ randomised control trial, $I V$ intervention, $C$ control, $N R$ not reported, $F / U$ follow-up, $S$ smoking, $N$ nutrition, $A$ alcohol, $P$ physical activity, $N R$ not reported

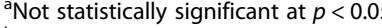

${ }^{\mathrm{b}}$ Significance testing not conducted

CPaper reported results separately for this clinician target

${ }^{\mathrm{d} C}$ Correctly reported from paper. Confidence intervals (1.3-2.0 vs $\left.1.4-2.0\right)$

e Note: only effect size results summarised. Group M and $95 \% \mathrm{CI}$ for IV and C groups at baseline, 6 - and 12-month follow-up reported but not summarised in table due to space constraints

fWIC refers to Special Supplemental Food Program for Women, Infants and Children 


\section{Intervention strategies}

One trial used one practice change intervention strategy [72], two trials used three strategies $[66,71]$, three used four strategies [67-69], and one used five [70]. Six of the seven trials reported utilising educational meetings as an intervention strategy $[66-69,71,72]$, four reported using patient resources $[66,67,69,71]$, and each of the following strategies was reported to be utilised by two trials: audit and feedback [69, 70]; patient-mediated intervention [68, 70]; educational outreach visits and academic detailing $[68,70]$; ongoing support $[68,70]$; distribution of educational materials [66, 67]; and reminders [67, 69]. One trial used local consensus processes [70]. The control condition consisted of usual care for three trials $[67,71,72]$, minimal intervention strategies in three (e.g. patient resources [66], audit and feedback [70], and patient-mediated intervention [68] and distribution of educational materials [68]), and was not specified in one trial [69].

\section{Clinician group receiving the intervention and data collection tools}

The nursing or allied health clinician groups that were the target of the practice change intervention for these trials were predominantly nurses (7/7 trials, including general practice nurses $[68,70,72]$, generalist community health nurses [67], registered nurses and licenced practical nurses [69], midwives [66], health visitors [72], and family planning counsellors, and WIC nurses counsellors [71]). Only one trial included allied health professionals (in addition to nurses) including dentists, dental hygienists, and mental health counsellors [71].

Clinician questionnaires were used to measure preventive care delivery in three trials $[67,68,71]$, with medical records audit [70], client questionnaires [72], client interviews [69], and a combination of client and clinician questionnaires also used [66].

\section{Behavioural risks addressed}

One trial focused on all four of the behavioural risks [67], with all others focusing on only one of these risks (five on smoking [66, 69-72] and one on alcohol overconsumption [68]).

\section{Preventive care practices}

The trial reporting preventive care provision regarding all four risks utilised a reduced model of care that focused on risk assessment, brief advice/assistance, and referral [67]. The five trials that focused on smoking only examined risk assessment $(4 / 5)[66,69,70,72]$, assessment of readiness to change (1/5) [69], advice (4/5) $[66,69-71]$, assistance $(4 / 5)[66,69-71]$, and arranging referral $(3 / 5)[66,70,71]$. The one trial that focused on only alcohol overconsumption examined risk assessment and advice [68]. For trials that addressed 'arranging referral', the variables of interest were referral to other service providers/support services for each of the four risks (including a quitline for smoking) in the multiple-risk trial [67], and referral to the following in the smoking-focused trials; a support group [71], a quit group [71], one-to-one telephone support [71], or discussing 'aftercare' [66].

\section{Risk of bias}

Table 3 provides a summary of judgements regarding the risk of bias at the outcome level for each trial. Overall, trial quality was difficult to assess given insufficient information reported regarding risk of bias classifications. Five trial authors supplied further information regarding unclear classifications upon contact [66-70]. All trials had at least one high risk of bias judgement, and as such, none were judged to be of high methodological quality.

Table 3 Summary of risk of bias in individual trials

\begin{tabular}{|c|c|c|c|c|c|c|c|}
\hline $\begin{array}{l}\text { Intervention trial } \\
\text { author, year, and } \\
\text { trial design }\end{array}$ & $\begin{array}{l}\text { Random } \\
\text { sequence } \\
\text { generation } \\
\text { (selection bias) }\end{array}$ & $\begin{array}{l}\text { Allocation } \\
\text { concealment } \\
\text { (selection bias) }\end{array}$ & $\begin{array}{l}\text { Blinding of } \\
\text { participants and } \\
\text { personnel } \\
\text { (performance bias) }\end{array}$ & $\begin{array}{l}\text { Blinding of } \\
\text { outcome } \\
\text { assessment } \\
\text { (detection bias) }\end{array}$ & $\begin{array}{l}\text { Incomplete } \\
\text { outcome data } \\
\text { (attrition bias) }\end{array}$ & $\begin{array}{l}\text { Selective } \\
\text { reporting } \\
\text { (reporting bias) }\end{array}$ & $\begin{array}{l}\text { Other } \\
\text { potential } \\
\text { sources } \\
\text { of bias }\end{array}$ \\
\hline $\begin{array}{l}\text { Bakker-(2003) [66]-Cluster RCT, } \\
\text { cross-sectional design } \\
\text { (post-test only). }\end{array}$ & Low & Unclear & Unclear & Unclear $^{a}$ & Low $^{a}$ & Unclear & High \\
\hline $\begin{array}{l}\text { Chan-(2013) [67]-Quasi- } \\
\text { experimental design }\end{array}$ & High & High & High & Low & High & Low & High \\
\hline Kaner-(2003) [68]-Cluster RCT & Low & Low & Low & High & Low & Unclear & High \\
\hline Katz-(2004) [69]-RCT & Low & Unclear & Unclear & Unclear & Low & Unclear & High \\
\hline Lennox-(1998) [72]-RCT & Low & Unclear & Low & Low & Low & High & High \\
\hline Moher-(2001) [70]-Cluster RCT & Low & Low & High & High & Low & Unclear & High \\
\hline Secker-Walker,-(2000) [71]-Non-RCT & Unclear & Unclear & Unclear & Unclear & High & High & High \\
\hline
\end{tabular}

${ }^{\mathrm{a}}$ Same risk of bias judgement for both classes of outcomes (clinician reported and client reported) 


\section{Results of trials}

\section{Intervention effect on preventive care delivery}

Of the seven trials, six conducted significance testing, including the multiple-risk trial [66-68, 70-72]. Trials reporting a significant increase in at least one variable included three $[66,71]$, four $[67,68]$, or five [70] of the following interventions strategies (from most to least frequent): educational meetings $[66-68,71]$, patient resources [66, 67, 71], patient-mediated intervention $[68,70]$, educational outreach visits and academic detailing $[68,70]$, ongoing support $[68,70]$, distribution of educational materials [66, 67], audit and feedback [70], reminders [67], and local consensus processes [70].

Smoking Of the four trials that sought to enhance the delivery of smoking assessment ('ask') [66, 67, 70, 72], two showed a positive effect for at least one measure of clinician assessment of client risk $[66,70]$ (the multi-risk trial found no effect). Of the three trials examining smoking brief advice $[66,67,71]$ (one examining a combined measure of brief advice and assistance) [67], two showed a positive effect of the intervention for at least one measure of brief advice [66, 67]. Of the two trials examining smoking assistance (specifically the provision of self-help materials [71] and setting quit dates [66, $71]$ ), both demonstrated a positive effect of the intervention for at least one measure of assistance [66, 71]. Lastly, of the three trials that sought to enhance arranging referral for smoking $[66,67,71]$, two demonstrated a positive effect of the intervention for at least one measure of the provision of referral (specifically discussing aftercare [66] and referral to a support group, quit group, and telephone support [71]). However the multirisk trial found no effect for smoking referral [67].

The studies reported multiple analyses for each care element, for example using different variables [66, 71], conducting different analyses for different data collection methods [66], or clinician subgroups [71, 72], using different statistical techniques [71], or examining different follow-up points $[67,71]$. Of the six analyses of smoking risk assessment conducted in four trials [66, 67, 70, 72], two demonstrated positive intervention effects $[66,70]$. Of the 15 brief advice analyses conducted in three trials $[66,67,71]$, four demonstrated positive intervention effects $[66,67]$. Of the 22 smoking assistance analyses conducted in two trials [66, 71], five demonstrated positive intervention effects [66, 71]. Of the 39 smoking referral analyses conducted in three trials $[66,67$, 71 , nine demonstrated positive intervention effects $[66,71]$.

Alcohol The following results were found for the two trials with a focus on alcohol preventive care provision $[67,68]$. Both trials included a measure of alcohol risk assessment and brief advice measures [67, 68]; one trial examined a combined measure of brief advice and assistance) [67] and only one trial showed a positive effect of the intervention on assessment and advice [68]. The trial examining all four risks simultaneously found no effect with regard to alcohol assessment or brief advice [67]. In the one trial examining arranging alcohol referral (the multi-risk trial), the intervention effect was not significant [67].

Inadequate nutrition and physical inactivity No trials focused solely on increasing care for inadequate nutrition and physical inactivity as independent risks. However, with regard to nutrition risk assessment, the multiple-risk trial demonstrated a positive effect of the intervention. With regard to nutrition brief advice and arranging referral, neither measure showed a significant intervention effect [67]. With regard to physical inactivity assessment and brief advice, this trial demonstrated a positive effect of the intervention [67]. However, with regard to arranging physical inactivity referral, there was no significant intervention effect [67].

\section{Discussion}

Seven intervention trials were located that described the effectiveness of strategies to increase the provision of preventive care regarding smoking, inadequate nutrition, alcohol overconsumption, or physical inactivity by primary care nurses or allied health clinicians. The trials were predominantly undertaken over 10 years ago (6/7), and only one included professionals other than nurses. While there was some evidence to indicate that practice change interventions for such clinicians may be effective in increasing the provision of smoking cessation care, this was limited given the small number of studies and the inconsistency of effect between and within trials. The effectiveness of interventions to increase care for alcohol overconsumption, inadequate nutrition, and physical inactivity and for multiple risks is unclear given the very small number of trials that examined care regarding these risk factors. Such conclusions are further qualified as no trials were judged to be of high methodological quality. Additional research is needed to determine the capacity of interventions to increase the provision of multiple elements of preventive care for these four priority behavioural risks by both nurses and allied health professionals in primary care settings.

The suggestion that interventions may be effective for smoking cessation care is based on the significant increase in at least one preventive care element reported in four of the five included trials that examined smoking and conducted significance testing. For the one trial that reported an effect size for smoking cessation care (17\%) [70], the effect size was consistent with past Cochrane 
reviews examining the effect of practice change strategies on health care practices more broadly [56-61]. In such Cochrane reviews, small to moderate improvements in care delivery were noted, with median adjusted $[56,58,61]$ or absolute [59] effect size differences ranging from $2 \%$ [59] to $6 \%$ [56] for categorical professional outcomes [56, 58, 59, 61] and from $1.3 \%$ [58] to $21 \%$ [61] for continuous outcomes [56, 58, 59, 61]. However, in the current review, the lack of consistency of the effectiveness of the multi-strategic approach within and between studies necessitates that conclusions regarding effectiveness on smoking care are made cautiously. When summaries are based on number of analyses undertaken for each care element, the results are less positive. For example, of the 39 smoking referral analyses conducted in three trials $[66,67,71]$, only seven demonstrated a positive effect of the intervention [66, 71]. We would conclude that the results are unclear.

Further, conclusions with regard to alcohol overconsumption, nutrition, and physical inactivity cannot be drawn. Only two trials examined preventive care regarding alcohol overconsumption. Such trials reported conflicting findings and suggested relatively small or no effects $[67,68]$. This differed to the modest effectiveness reported in the aforementioned systematic reviews based on a larger number of trials (11-12) that examined effect of practice change on delivery of preventive care for alcohol within primary care settings (care predominantly by physicians) $[63,64]$. With regard to increasing inadequate nutrition and physical inactivity, only one trial was identified and reported variable effect by risk and care element.

The findings indicate the need for further investigation of intervention approaches that may result in an increased prevalence of care across risk factors and care elements [73]. All trials in the current review, bar one [72], used a multi-strategic intervention including between three and five practice change strategies. While such an approach is supported by other studies and reviews recommending the inclusion of multiple practice change strategies within intervention trials $[38,62-64,74-79]$, an overview of systematic reviews evaluating the effectiveness of multistrategic interventions in changing health care professional's behaviour in clinical settings found no compelling evidence that such interventions are more effective than single strategy interventions [80]. Strategies implemented might be informed by barriers to care delivery at the client, clinician, and system level in light of review evidence supporting the effectiveness of tailoring intervention strategies to determinants of practice (barriers, obstacles, enablers, and facilitators) [81]. In regard to smoking cessation care specifically, a multi-strategic approach is recommended by various clinical guidelines [1, 7, 38]. For example, the United States Treating Tobacco Use and
Dependence Clinical Practice Guidelines recommend the implementation of a tobacco user identification system; provision of education, resources, and feedback to promote provider intervention; dedicate staff to provide tobacco dependence treatment, and assess its delivery in staff performance evaluations; promotion of hospital policies that support and provide inpatient tobacco dependence services; and the inclusion of tobacco dependence treatments as paid or covered services in all subscribers or members of health insurance packages [38].

The findings of this review should be considered in light of a number of limitations. The generalisability of the review conclusions to allied health clinicians is limited as only one trial included allied health clinicians, and this trial also included nurses. Similarly, generalisability across primary care settings is limited as most included trials were conducted in a limited range of settings, predominantly primary care practices/clinics/ general practices [68-72], with few or no studies in settings such as community health services, Health Maintenance Organisations, Primary Care Trusts, or mobile nursing services. Additionally, the trial results are predominantly from studies published over 10 years ago and hence more current evidence regarding the effectiveness of practice change interventions for primary care nurses and allied health professionals is unclear. Furthermore, as a consequence of inferring 5 A's terminology for studies included in the review, definitions of what constituted the same element of care could vary and hence may account for some of the variability between and within studies on such measures. Also, although the current review utilised a broad search strategy, only trials published in journals within the included databases were located [65]. Finally, the search was also limited by having only one author conducting the title and abstract review.

\section{Conclusions}

The current review indicated that there is little evidence on the effectiveness of practice change interventions for primary care nurses and allied health professionals. The small number of trials focused on care for smoking shows intervention effects to be inconsistent between and within studies. Evidence for the effectiveness of interventions to increase care for alcohol overconsumption, inadequate nutrition, and physical inactivity and for multiple risks is also inconclusive as they were examined in an even more limited number of trials with inconsistent findings. There is a need for further research with regard to effective interventions to increase preventive care by nurses and in particular allied health professionals in primary care settings. Such research could examine a range of care elements regarding smoking, alcohol overconsumption, inadequate nutrition, physical inactivity, and for multiple risks. 


\section{Additional file}

Additional file 1: Table S1. The Cochrane Collaboration's tool for assessing risk of bias [65]. Table S2. Criteria for judging risk of bias in the 'Risk of bias' assessment tool [65]. (DOCX 53 kb)

\section{Competing interests}

The authors declare that they have no competing interests.

\section{Authors' contributions}

KM led the development of the manuscript, undertook the literature search, and summarised and interpreted the data. Authors MF, LC, JW, and KM contributed to the conception and design of the systematic review, interpretation of the findings, and drafting and revision of the manuscript. Authors KB and PW contributed to the risk of bias analyses, interpretation of the data, and the revision of the manuscript. All authors approved the final version.

\section{Acknowledgements}

This research was undertaken with funding from The University of Newcastle, Australia; Population Health, Hunter New England Local Health District; the National Health and Medical Research Council $(I D=1016650)$ and infrastructure support from the Hunter Medical Research Institute. The funding organisations had no role in the initiation, design and conduct of the study; in the collection, analysis, and interpretation of the data; or in the preparation, review, or approval of the manuscript.

\section{Author details}

${ }^{1}$ Population Health, Hunter New England Local Health District, Booth Building, Wallsend Health Services, Longworth Avenue, Wallsend, NSW 2287, Australia. ${ }^{2}$ Faculty of Health, The University of Newcastle, University Drive, Callaghan, NSW 2308, Australia. ${ }^{3}$ Hunter Medical Research Institute, Clinical Research Centre, Lot 1 Kookaburra Circuit, New Lambton Heights, NSW 2305, Australia. ${ }^{4}$ School of Psychology, Faculty of Science and Information Technology, The University of Newcastle, University Drive, Callaghan, NSW 2308, Australia. ${ }^{5}$ Postal address: Locked Bag 10, Wallsend, NSW 2287, Australia.

Received: 31 July 2015 Accepted: 16 March 2016

\section{Published online: 06 April 2016}

\section{References}

1. The Royal Australian College of General Practitioners. Guidelines for preventive activities in general practice (the red book). 8th ed. Melbourne: The Royal Australian College of General Practitioners; 2012.

2. U.S.Department of Health and Human Services. The guide to clinical preventive services 2014: recommendations of the U.S. Preventive Services Task Force. Rockville, MD: Agency for Healthcare Research and Quality; 2014

3. National Institute for Health and Clinical Excellence (NICE). Brief interventions and referral for smoking cessation: NICE public health guidance 1.guidance.nice.org.uk/ph1. 2006. Accessed 1 Sep 2014.

4. National Institute for Health and Clinical Excellence (NICE). Alcohol-use disorders: diagnosis, assessment and management of harmful drinking and alcohol dependence. NICE public health guidance 115. 2011. Accessed 1 Sep 2014

5. National Institute for Health and Clinical Excellence (NICE). Physical activity: brief advice for adults in primary care. NICE public health guidance 44 2013. Accessed 1 Sep 2014

6. National Institute for Health and Clinical Excellence (NICE). Recommendations about diet for primary care and community health services. NICE diet pathway. 2014. Accessed 1 Sep 2014.

7. National Preventative Health Taskforce. Australia: the healthiest country by 2020. National preventative health strategy—overview. Canberra: National Preventative Health Taskforce; 2009.

8. Ministry of Health. New Zealand smoking cessation guidelines. Wellington: Ministry of Health; 2007.

9. Prochaska JJ, Spring B, Nigg CR. Multiple health behavior change research: an introduction and overview. Prev Med. 2008;46:181-8.

10. World Health Organisation (WHO). The World Health Report: reducing risks, promoting healthy lifestyle. Geneva: WHO; 2002.

11. Australian Institute of Health and Welfare (AlHW). Australia's health 2010. Canberra: AlHW; 2010.
12. Owen A. Community Health: The evidence base. A report for the NSW Community Health Review. Wollongong: Centre for Health Service Development, University of Wollongong; 2010.

13. Smith K, Bazini-Barakat N. A public health nursing practice model: melding public health principles with the nursing process. Public Health Nurs. 2003;20:42-8.

14. Whitlock EP, Orleans CT, Pender N, Allan J. Evaluating primary care behavioral counseling interventions: an evidence-based approach. Am J Prev Med. 2002;22:267-84.'

15. National Preventative Health Taskforce. Australia: the healthiest country by 2020. A discussion paper. Canberra: National Preventative Health Taskforce; 2008.

16. Chan B, Laws R, Williams A, Davies G, Fanaian M, Harris $M$, et al. Is there scope for community health nurses to address lifestyle risk factors? the community nursing SNAP trial. BMC Nurs. 2012:11:4.

17. The Royal Australian College of General Practitioners. Guidelines for preventative activities in general practice. South Melbourne, Victoria: The Royal Australian College of General Practitioners; 2009.

18. Goldstein MG, Whitlock EP, DePue J. Multiple behavioral risk factor interventions in primary care. Summary of research evidence. Am J Prev Med. 2004:27:61-79.

19. Rice VH, Stead LF. Nursing interventions for smoking cessation. Cochrane Database Syst Rev. 2008;1:CD001188. doi:10.1002/14651858.CD001188.pub3.

20. Stead LF, Buitrago D, Preciado N, Sanchez G, Hartmann-Boyce J, Lancaster T. Physician advice for smoking cessation. Cochrane Database Syst Rev. 2013;5:CD000165. doi:10.1002/14651858.CD000165.pub4.

21. Brunner E, Rees R, Ward K, Burke M, Thorogood M. Dietary advice for reducing cardiovascular risk. Cochrane Database Syst Rev. 2007;4:CD002128. doi:10.1002/14651858.CD002128.pub3.

22. Kaner EF, Beyer F, Dickinson HO, Pienaar E, Campbell F, Schlesinger C, et al. Effectiveness of brief alcohol interventions in primary care populations. Cochrane Database Syst Rev. 2007:2:CD004148. doi:10.1002/14651858. CD004148.pub3

23. Foster $\mathrm{C}$, Hillsdon $\mathrm{M}$, Thorogood M, Kaur A, Wedatilake T. Interventions for promoting physical activity. Cochrane Database Syst Rev. 2005;1:CD003180. doi:10.1002/14651858.CD003180.pub2.

24. Ashenden R, Silagy C, Weller D. A systematic review of the effectiveness of promoting lifestyle change in general practice. Fam Pract. 1997:14:160-75.

25. Fiore MC, Bailey WC, Cohen SJ, Dorfman SF, Goldstein MG, Gritz ER. Treating tobacco use and dependence: clinical practice guideline. Rockville (MD): US Department of Health and Human Services. Public Health Service; 2000.

26. Kottke TE, Battista RN, DeFriese GH, Brekke ML. Attributes of successful smoking cessation interventions in medical practice: a meta-analysis of 39 controlled trials. J Am Med Assoc. 1988;259:2883-9.

27. Mullen PD, Simons-Morton DG, Ramirez G, Frankowski RF, Green LW, Mains DA. A meta-analysis of trials evaluating patient education and counseling for three groups of preventive health behaviors. Patient Educ Couns. 1997;32:157-73.

28. Puschel $K$, Thompson B, Coronado G, Huang Y, Rivera S. Effectiveness of a brief intervention based on the $5 \mathrm{~A}$ model for smoking cessation at the primary care level in Santiago, Chile. Health Promot Int. 2008;23:240-50.

29. Wilk Al, Jensen NM, Havighurst TC. Meta-analysis of randomized control trials addressing brief interventions in heavy alcohol drinkers. J Gen Intern Med. 1997:12(5):274-83.

30. Bien TH, Miller WR, Tonigan JS. Brief interventions for alcohol problems: a review. Addiction. 1993:88:315-36.

31. Bertholet N, Daeppen JB, Wietlisbach V, Fleming M, Burnand B. Reduction of alcohol consumption by brief alcohol intervention in primary care: systematic review and metaanalysis. Arch Intern Med. 2005;165:986-95.

32. Brunner E, White I, Thorogood M, Bristow A, Curle D, Marmot M. Can dietary interventions change diet and cardiovascular risk factors? A meta-analysis of randomized controlled trials. Am J Public Health. 1997;87:1415-22.

33. Smith B. Promotion of physical activity in primary health care: update of the evidence on interventions. J Sci Med Sport. 2004;7:67-73.

34. Simon-Morton D, Calfas K, Oldenburg BBN, Burton N. Effects of interventions in health care settings on physical activity or cardiorespiratory fitness. Am J Prev Med. 1998:15:413-30.

35. Australian Government. A healthier future for all Australians. Final report Canberra: Australian Government; 2009.

36. New South Wales (NSW) Health. Integrated primary and community health policy 2007-2012. North Sydney, NSW, Australia: NSW Health; 2006.

37. U.S.Department of Health and Human Services. The guide to clinical preventive services 2008: recommendations of the U.S. Preventive Services Taskforce. Rockville, MD: Agency for Healthcare Research and Quality; 2008. 
38. Fiore MC, Jaen CR, Baker TB. Treating tobacco use and dependence: 2008 update. Clinical practice guideline. Rockville, MD: U.S. Department of Health and Human Services; 2008.

39. Laws RA, Davies GP, Williams A, Eames-Brown R, Amoroso C, Harris M. Community health risk factor management research project. Final report February 2008. A feasibility study. Sydney: University of NSW; 2008.

40. Eagar K, Owen A, Cranny C, Samsa P, Thompson C. Community health: the state of play in New South Wales. A report for the NSW Community Health Review. Wollongong: Centre for Health Service Development, University of Wollongong; 2008.

41. Laws RA, Jayasinghe UW, Harris MF, Williams AM, Davies GP, Kemp LA Explaining the variation in the management of lifestyle risk factors in primary health care: a multilevel cross sectional study. BMC Public Health. 2009;9:165.

42. Hung DY, Shelley DR. Multilevel analysis of the chronic care model and 5A services for treating tobacco use in urban primary care clinics. Health Serv Res. 2009;44:103-27.

43. Dauenhauer JA, Podgorski CA, Karuza J. Prescribing exercise for older adults: a needs assessment comparing primary care physicians, nurse practitioners, and physician assistants. Gerontol Geriatr Educ. 2006;26:81-99.

44. Brule J, Abboud C, Deschambault E. Smoking cessation counselling practices among Quebec optometrists: evaluating beliefs, practices, barriers and needs. Clin Exp Optom. 2012;95:599-605.

45. Marley JV, Nelson C, O'Donnell V, Atkinson D. Quality indicators of diabetes care: an example of remote-area Aboriginal primary health care over 10 years. Med J Aust. 2012;197:2012

46. Akpanudo SM, Price JH, Jordan T, Khuder S, Price JA. Clinical psychologists and smoking cessation: treatment practices and perceptions. J Community Health. 2009;34:461-71.

47. Dillman CJ, Shields CA, Fowles JR, Perry A, Murphy RJL, Dunbar P. Including physical activity and exercise in diabetes management: diabetes educators' perceptions of their own abilities and the abilities of their patients. Can J Diabetes. 2010;34:218-26.

48. Hopkins SC, Lenz ER, Pontes NM, Lin SX, Mundinger MO. Context of care or provider training: the impact on preventive screening practices. Prev Med. 2005:40:718-24

49. Horvath K, Eastman M, Prosser R, Goodroad B, Worthington L. Addressing smoking during medical visits: patients with human immunodeficiency virus. Am J Prev Med. 2012;43:21.

50. Douglas F, Torrance N, Van TE, Meloni S, Kerr A. Primary care staff's views and experiences related to routinely advising patients about physical activity. A questionnaire survey. BMC Public Health. 2006;6:138.

51. Si D, Bailie RS, Dowden M, O'Donoghue L, Connors C, Robinson GW, et al. Delivery of preventive health services to Indigenous adults: response to a systems-oriented primary care quality improvement intervention. Med J Aust. 2007;187:453-7.

52. Glover M, Paynter J, Bullen C, Kristensen K. Supporting pregnant women to quit smoking: postal survey of New Zealand general practitioners and midwives' smoking cessation knowledge and practices. N Z Med J. 2008;121:53-64.

53. Runciman $\mathrm{P}$, Watson $\mathrm{H}$, Mclntosh J, Tolson D. Community nurses' health promotion work with older people. J Adv Nurs. 2006;55:46-57.

54. McElwaine KM, Freund M, Campbell E, Knight J, Bowman J, Doherty E et al. The delivery of preventive care to clients of community health services. BMC Health Serv Res 2013;13. doi:10.1186/1472-6963-13-167.

55. McElwaine KM, Freund M, Campbell EM, Slattery C, Wye PM, Lecathelinais C, et al. Clinician assessment, advice and referral for multiple health risk behaviours: prevalence and predictors of delivery by primary health care nurses and allied health professionals. Patient Educ Couns. 2013;94:193-201.

56. Forsetlund L, Bjorndal A, Rashidian A, Jamtvedt G, O'Brien MA, Wolf F, et al. Continuing education meetings and workshops: effects on professional practice and health care outcomes. Cochrane Database Syst Rev. 2009:2:CD003030.

57. Renders CM, Valk GD, Griffin S, Wagner EH, Eijk JT, Assendelft WJ. Interventions to improve the management of diabetes mellitus in primary care, outpatient and community settings. Cochrane Database Syst Rev. 2001;1:CD001481.

58. Ivers N, Jamtvedt G, Flottorp S, Young JM, Odgaard-Jensen J, French SD, et al. Audit and feedback: effects on professional practice and healthcare outcomes. Cochrane Database Syst Rev. 2012;6:CD000259. doi:10.1002/ 14651858.CD000259.pub3.
59. Giguère A, Légaré F, Grimshaw J, Turcotte $S$, Fiander $M$, Grudniewicz A, et al. Printed educational materials: effects on professional practice and healthcare outcomes. Cochrane Database Syst Rev. 2012;10:CD004398. doi:10.1002/14651858.CD004398.pub3.

60. Flodgren G, Eccles MP, Shepperd S, Scott A, Parmelli E, Beyer FR. Printed educational materials: effects on professional practice and healthcare outcomes. Cochrane Database Syst Rev. 2011;7:CD009255. doi:10.1002/ 14651858.CD009255.

61. O'Brien MA, Rogers S, Jamtvedt G, Oxman AD, Odgaard-Jensen J, Kristoffersen DT, et al. Educational outreach visits: effects on professional practice and health care outcomes. Cochrane Database Syst Rev. 2007;4:CD000409.

62. Papadakis S, McDonald P, Mullen KA, Reid R, Skulsky K, Pipe A. Strategies to increase the delivery of smoking cessation treatments in primary care settings: a systematic review and meta-analysis. Prev Med. 2010;51:199-213.

63. Anderson P, Laurant M, Kaner E, Wensing M, Grol R. Engaging general practitioners in the management of hazardous and harmful alcohol consumption: results of a meta-analysis. J Stud Alcohol. 2004:65:191-9.

64. Nilsen P, Aalto M, Bendtsen P, Seppa K. Effectiveness of strategies to implement brief alcohol intervention in primary healthcare. A systematic review. Scand J Prim Health Care. 2006;24:5-15.

65. Higgins JPT, Green S. Cochrane Handbook for Systematic Reviews of Interventions. Version 5.1.0 [updated March 2011]. In: Higgins JPT, Green S, editors. The Cochrane Collaboration, 2011. 2011.

66. Bakker MJ, Mullen PD, De Vries H, Van Breukelen G. Feasibility of implementation of a Dutch smoking cessation and relapse prevention protocol for pregnant women. Patient Educ Couns. 2003;49:35-43.

67. Chan BC, Jayasinghe UW, Christl B, Laws RA, Orr N, Williams A, et al. The impact of a team-based intervention on the lifestyle risk factor management practices of community nurses: outcomes of the community nursing SNAP trial. BMC Health Serv Res. 2013;13:54.

68. Kaner E, Lock C, Heather N, McNamee P, Bond S. Promoting brief alcohol intervention by nurses in primary care: a cluster randomised controlled trial. Patient Educ Couns. 2003;51:277-84.

69. Katz DA, Brown RB, Muehlenbruch DR, Fiore MC, Baker TB. AHRQ smoking cessation guideline study group: implementing guidelines for smoking cessation: comparing the efforts of nurses and medical assistants. Am J Prev Med. 2004;27:411-6.

70. Moher M, Yudkin P, Wright L, Turner R, Fuller A, Schofield T, et al. Cluster randomised controlled trial to compare three methods of promoting secondary prevention of coronary heart disease in primary care. Br Med J. 2001;322:1338.

71. Secker-Walker RH, Dana GS, Solomon LJ, Flynn BS, Geller BM. The role of health professionals in a community-based program to help women quit smoking. Prev Med. 2000;30:126-37.

72. Lennox AS, Bain N, Taylor RJ, McKie L, Donnan PT, Groves J. Stages of change training for opportunistic smoking intervention by the primary health care team. Part I: randomised controlled trial of the effect of training on patient smoking outcomes and health professional behaviour as recalled by patients. Health Educ J. 1998:57:140-9.

73. McElwaine KM, Freund M, Campbell E, Knight J, Wolfenden L, Bartlem K, et al. Increasing preventive care by primary care nursing and allied health clinicians: a non-randomized, controlled trial. Am J Prev Med. 2014;47:424-34.

74. Curry SJ, Keller PA, Orleans CT, Fiore MC. The role of health care systems in increased tobacco cessation. Annu Rev Public Health. 2008;29:411-28.

75. Dijkstra R, Wensing M, Thomas R, Akkermans R, Braspenning J, Grimshaw J et al. The relationship between organisational characteristics and the effects of clinical guidelines on medical performance in hospitals, a meta-analysis. BMC Health Serv Res 2006, 6: DOl:10.1186/1472-6963-6-53.

76. Grimshaw J, Eccles M, Thomas R, MacLennan G, Ramsay C, Fraser C, et al. Toward evidence-based quality improvement. Evidence (and its limitations) of the effectiveness of guideline dissemination and implementation strategies 1966-1998. J Gen Intern Med. 2006;21:14-20.

77. Freund M, Campbell E, Paul C, Sakrouge R, McElduff P, Walsh R, et al. Increasing smoking cessation care provision in hospitals: a meta-analysis of intervention effect. Nicotine Tob Res. 2009;11:650-62.

78. Jamtvedt G, Young JM, Kristoffersen DT, Thomson O'Brien MA, Oxman AD. Audit and feedback: effects on professional practice and health care outcomes. Cochrane Database Syst Rev. 2006;2:CD000259. doi:10.1002/ 14651858.CD000259.pub2. 
79. Grol R, Wensing M, Hulscher M, Eccles M. Theories on implementation of change in healthcare. In: Grol R, Wensing M, Eccles M, editors. Improving patient care: the implementation of change in clinical practice. London: Elsevier; 2004.

80. Squires JE, Sullivan K, Eccles MP, Worswick J, Grimshaw JM. Are multifaceted interventions more effective than single-component interventions in changing health-care professionals' behaviours? An overview of systematic reviews. Imp Sci. 2014;152.

81. Baker R, Camosso-Stefinovic J, Gillies C, Shaw EJ, Cheater F, Flottorp S, et al. Tailored interventions to address determinants of practice. Cochrane Database Syst Rev. 2015:4:CD005470.

82. Grimshaw J, McAuley LM, Bero LA, Grilli R, Oxman AD, Ramsay C, et al. Systematic reviews of the effectiveness of quality improvement strategies and programmes. Qual Saf Health Care. 2003;12:298-303.

Submit your next manuscript to BioMed Central and we will help you at every step:

- We accept pre-submission inquiries

- Our selector tool helps you to find the most relevant journal

- We provide round the clock customer support

- Convenient online submission

- Thorough peer review

- Inclusion in PubMed and all major indexing services

- Maximum visibility for your research

Submit your manuscript at www.biomedcentral.com/submit
Biomed Central 